\title{
Increased Expression of eEF1A2 and PI3K-Akt Signaling Pathway Genes Promotes The Progression of Cervical Cancer
}

\section{Huizhen Xin}

Department of Biochemistry and Molecular Biology, School of Medicine, Shihezi University Huan Pan

Department of Biochemistry and Molecular Biology, School of Medicine, Shihezi University

\section{Xiangyi Zhe}

Department of Biochemistry and Molecular Biology, School of Medicine, Shihezi University

\section{Chunhe Zhang}

Department of Biochemistry and Molecular Biology, School of Medicine, Shihezi University

\section{Hongtao Li}

Department of Biochemistry and Molecular Biology, School of Medicine, Shihezi University

\section{Weinan Zheng}

Department of Anatomy and Histology and Embryology, Development and Regeneration Key Lab of Sichuan Province, Chengdu Medical College

\section{Renfu Shao}

School of Science and Engineering, Genecology Research Centre, University of the Sunshine Coast, Sippy Downs, Queensland

\section{Dongmei Li}

Department of Biochemistry and Molecular Biology, School of Medicine, Shihezi University

\section{Zemin Pan ( $\nabla$ panteacher89@sina.com )}

Department of Biochemistry and Molecular Biology, School of Medicine, Shihezi University

\section{Research Article}

Keywords: cervical cancer, eEF1A2, PI3K-Akt signaling pathway, HPV16 L1

Posted Date: February 4th, 2021

DOI: https://doi.org/10.21203/rs.3.rs-144942/v1

License: (c) (i) This work is licensed under a Creative Commons Attribution 4.0 International License. Read Full License 


\section{Increased expression of eEF1A2 and PI3K-Akt signaling pathway genes promotes the}

progression of cervical cancer

Huizhen Xin ${ }^{\dagger 1}$, Huan Pan ${ }^{\dagger 1}$, Xiangyi Zhe ${ }^{1}$, Chunhe Zhang ${ }^{1}$, Hongtao $\mathrm{Li}^{1}$, Weinan Zheng ${ }^{2}$, Renfu Shao ${ }^{3}$, Dongmei $\mathrm{Li}^{*}{ }^{1}$ and Zemin $\mathrm{Pan}^{* 1}$

*Correspondence: Zemin Pan (panteacher89@sina.com); Dongmei Li (lidong_abc@126.com) Department of Biochemistry and Molecular Biology, School of Medicine, Shihezi University, Shihezi, China.

${ }^{1}$ Department of Biochemistry and Molecular Biology, School of Medicine, Shihezi University, Xinjiang Endemic and Ethnic Disease and Education Ministry Key Laboratory, Shihezi, Xinjiang, 832002, China

${ }^{2}$ Department of Anatomy and Histology and Embryology, Development and Regeneration Key Lab of Sichuan Province, Chengdu Medical College, Chengdu, Sichuan, 610000, China ${ }^{3}$ School of Science and Engineering, Genecology Research Centre, University of the Sunshine Coast, Sippy Downs, Queensland, 4556, Australia

"These authors contributed equally: Huizhen Xin, Huan Pan

\section{Abstract}

Objective: This study sought to explore the mRNA and protein expression levels of eukaryotic translation elongation factor 1 alpha 2 (eEF1A2) and members of the PI3K-Akt signaling pathway in the context of cervical cancer. We sought to clarify the expression of eEF1A2, PI3K, Akt during cervical cancer tumorigenesis and development.

Methods: Samples from 72 cases of cervical cancer were collected, as well as 46 cases of cervical intraepithelial neoplasia (CIN), which reflects the continuous process of cervical cancer development, divided into CIN I, CIN II, CIN III, and 40 cases of chronic cervicitis. qRT-PCR was to detect the mRNA levels of eEF1A2, PI3K, and Akt, with $\beta$-actin used as an internal control. eEF1A2, PI3K, p-Akt protein levels in cervical cancer, CIN, and chronic cervicitis tissues were 
detected by immunohistochemical. eEF1A2, PI3K and p-Akt protein expression levels in HeLa, $\mathrm{SiHa}$, and human umbilical vein endothelial cells were detected by western blot.

Results: qRT-PCR results showed that the level of mRNA expression of eEF1A2, PI3K, and Akt was higher in cervical cancer tissues than that in normal cervical tissues. Immunohistochemistry results showed that the eEF1A2, PI3K, and p-Akt protein levels were higher in cervical cancer tissues than in cervical chronic cervicitis tissues. The differences were statistically significant $(\mathrm{P}<$ 0.05). The expression of eEF1A2, PI3K, and p-Akt protein was higher in HeLa and SiHa cervical cell lines than that in normal epithelial cells.

Conclusion: Together, these results suggest that the aberrant expression of eEF1A2, PI3K, and Akt may play a role in cervical cancer development and tumorigenesis.

Keywords: cervical cancer; eEF1A2; PI3K-Akt signaling pathway; HPV16 L1

\section{Background}

Cervical cancer is the second most common malignant tumor among females worldwide after breast cancer[1]. It is one of the major malignant tumors threatening the health of women, and it is often lethal. HPV plays an indispensable role in the early stages of cervical cancer. Among HPV viruses, HPV16 has been identified as being closely associated with the incidence of cervical cancer[2]. Today, high-risk HPV infection is an important factor in cervical intraepithelial neoplasia and cervical cancer, and clinical detection of high-risk HPV infections has become an effective diagnostic marker of cervical cancer.

In a previous study, we observed that eukaryotic protein elongation factor 1A (eukaryotic protein elongation factor 1 alpha 2, eEF1A2) and v-Akt murine thymoma viral oncogene homolog 1(Akt1), also known as protein kinase B (PKB), mRNA expression levels are increased in cervical 
cancer tissues[3]. Therefore, we hypothesized that the abnormal expression of eEF1A2 in cervical cancer tissue may affect the development and occurrence of cervical cancer. eEF1A2 is normally expressed only in the myocardium, brain and skeletal muscle of mammals, but is found to be abnormally highly expressed in a variety of malignant tumors. eEF1A2 is considered to be an oncogene, as abnormally high expression levels of eEF1A2 can not only result in tumor cell proliferation, but can also promote tumor invasion and metastasis $[4,5]$.

The eEF1A2 gene is located on chromosome 20q13.3, and has a full length of 11304bp. It codes for the protein eEFlA2, which has a molecular weight of $54 \mathrm{kDa}$. eEFlA2 is known to play an important role in the translation and extension of proteins. During the peptide chain extension phase, eEF1A2 and GTP produce a complex that binds to aminoacyl-tRNA, which is transferred onto the ribosome A site to extend the peptide chain, so as to ensure the accuracy and speed of protein translation.

The phosphatidylinositol-3-kinase (PI3K)-Akt signal transduction pathway plays an important role in the occurrence and development multiple types of tumors. Some studies have directly linked the PI3K-Akt signaling pathway to the occurrence and development of cervical cancer[6]. PI3K is a proto-oncogene, which is divided into three types according to the structure of the substrate[7]; one of the most widely studied is PI3K I, which can be activated by receptors on the cell surface, and is composed of a catalytic subunit and a regulatory subunit. Akt is a serine/threonine protein kinase encoded by the proto-oncogene c-Akt. Akt acts as an intracellular second messenger: when it is phosphorylated it activates a cascade which regulates processes such as cellular differentiation, migration, and apoptosis[8].

In this study, we detected the expression of HPV16 L1 protein in chronic cervicitis, CIN, and 
cervical cancer tissue. Additionally, we detected the mRNA expression levels of eEF1A2 and genes related to the PI3K-Akt signal transduction pathway in cervical cancer tissues, so as to understand the role of these genes in the occurrence and development of cervical cancer. By detecting the protein expression levels of eEF1A2 and other members of the PI3K-Akt signal transduction pathway in cervical cancer, we have both clarified the role of these genes in the occurrence and development of cervical cancer, as well as provided a reference value for the clinical diagnosis and pathological grading of cervical cancer.

\section{Methods and Materials}

Sample collection

Samples used in this study were collected between June 2012 and December 2014 at the First Affiliated Hospital of Shihezi University and the Xinjiang Korla Bazhou Hospital for Medical Treatment. Tissue samples were collected from 72 cases of cervical cancer, 46 cases of cervical intraepithelial neoplasia, and 40 cases of chronic cervicitis (supplementary Table 1). Tissue samples were embedded in paraffin for immunohistochemical experiments, and tissue sections were made and stored at $4{ }^{\circ} \mathrm{C}$. The median age of patients was 45 years old, ranging from 25 years old to 72 years old. Fresh tissue samples from ten patients with cervical cancer as well as normal cervical tissue from ten patients were collected for qRT-PCR experiments. The median age of these patients was 53 years old, ranging from 34 years old to 73 years old. All specimens were diagnosed by two experienced pathologists, and all patients provided written informed consent. The specimens were collected and stored at $-80^{\circ} \mathrm{C}$.

\section{Fluorescent real-time quantitative PCR}

Total RNA was extracted from cervical tissue samples using Trizol (Invitrogen, USA), according 
to the manufacturer's instructions. Extracted RNA was stored at $-80^{\circ} \mathrm{C}$ until use. Reverse transcription was performed using the Revert Aid First Strand cDNA Synthesis Kit (Life Technologies, USA), and the extracted DNA was stored at $-20^{\circ} \mathrm{C}$ until use. Fluorescent qRT- PCR was performed using a SYBR Green PCR Kit (QIAGEN, Germany). $\beta$-actin was used as the internal reference gene. The expression of eEF1A2, PIK3CA, and Akt1 was detected, and each sample was tested three times.

\section{Primer design and synthesis}

Based on the full-length sequence of human eEF1A2, PIK3CA, and Akt1 in the NCBI Genbank, primers were designed using Primer 5 software, and synthesized by Shanghai Biological Engineering Co. Ltd. (China, Table 1).

\section{Immunohistochemical staining}

The immunohistochemical staining two-step method was performed according to the manufacturer's instructions. As a negative control, Phosphate buffer solution (PBS) was substituted for primary antibody. The results were interpreted using a double-blinded method: two pathology experts blindly and independently assessed the specimens, and the average of the two assessments was taken to compute the final result. The calculation of positive intensity was determined by the product of the percentage of positive cells and the staining intensity of the cells (supplementary Table 2).

\section{Cell culture}

Human cervical carcinoma cell lines ( $\mathrm{SiHa}$ and HeLa) were maintained in our laboratory, and cultured in HIGH-DEME (Gibco, USA) media supplemented with $10 \%$ fetal bovine serum (SiJiQing, China), $100 \mathrm{U} / \mathrm{ml}$ penicillin, and $50 \mu \mathrm{g} / \mathrm{ml}$ streptomycin. Cells were maintained in 
incubators with a humidified atmosphere of $5 \% \mathrm{CO}_{2}$ and $95 \%$ air at $37^{\circ} \mathrm{C}$.

siRNA transfection

The SiHa and Hela cells were seeded into 6-well plates. si-eEF1A2 or negative control siRNA

(both purchased from GenePharma, Shanghai,China) were transfected using FuGENE HD

transfection reagent (Promega, USA) according to the manufacturer's instructions. After $48 \mathrm{~h}$ of transfection, cells were collected, siRNA efficiency was confirmed, and further functional assays were performed. The sequence of si-eEF1A2 was as follows:

si-eEF1A2-1:AATGCGGAGGTATTGACAAAA;

si-eEF1A2-2:TACGACGAGATCGTCAAGGAA.

\section{Cell proliferation assay}

SiHa and HeLa cells were seeded into 96-well plates, following $24 \mathrm{~h}$ of transfection in 6-well plates (Corning, NY, USA), at a concentration of 3000 cells/well. The relative number of viable cells was measured using a Cell Counting Kit-8 (CCK-8) kit (Dojindo Laboratories, Japan) at 24, 48, and $72 \mathrm{~h}$. Proliferation was assessed by measuring the absorbance at $450 \mathrm{~nm}$ using a microplate reader (Bio-Rad, USA).

\section{Colony formation assay}

SiHa cells and HeLa cells were seeded and transfected with si-eEF1A2 or negative control siRNA in a 6-well plate. After $24 \mathrm{~h}$, transfected cells were seeded into another 6-well plate at a concentration of 1000 cells/well, followed by culture for an additional 14 days. Cells were then fixed in methanol for 15 min followed by staining with $0.1 \%$ crystal violet for 15 min. Manual counting of more than 50 cell clones was performed in three independent experiments.

\section{Western blotting}


Cells were lysed in RIPA buffer, and the lysate was centrifuged at $12000 \mathrm{rpm}$ for $10 \mathrm{~min}$. Extracted proteins were transferred to polyvinylidene fluoride membranes by SDS-PAGE. Membranes were blocked with 5\% skim milk for $2 \mathrm{~h}$ followed by incubation for $16 \mathrm{~h}$ with anti-eEF1A2 rabbit monoclonal antibody (1:100; Biosynthesis, Beijing, China). Membranes were then washed three times in TBST, followed by incubation for $2 \mathrm{~h}$ with secondary antibody (1:10000; Zhongshang Jinqiao Biotechnology Co., Ltd., Beijing, China.) The protein strips are then developed and fixed under red light conditions in the darkroom: the western blotting luminol reagent solution $\mathrm{A}$ is mixed with the solution $\mathrm{B}$ in a ratio of 1:1 and uniformly added to the PVDF membrane; After the film is attached to the PVDF for 1 minute, it is placed in a developing solution for development, and after a clear protein band appears, it is then fixed in a fixing solution; Finally, take a picture with the imaging system.

\section{Statistical analysis}

SPSS19.0 statistical software was used to analyze and process data. At least three independent repeats were performed for each experiment. All values are expressed as the mean \pm standard deviation. Comparisons between two groups were analyzed using a Student's t-test. Correlation tests were performed using a McNemar analysis. The relationship between protein expression and the pathological characteristics of the tissue samples was analyzed using a chi-squared test. $P<$ 0.05 was considered statistically significant.

\section{Statement}

It is hereby declared that the experiments on human specimens involved in this study are conducted in strict accordance with relevant guidelines and regulations.

\section{Results}


Expression of HPV16 L1 protein in cervical cancer tissue

Immunohistochemical results are displayed in Figure 1. The expression intensity of HPV16 L1 in cervical cancer tissues was significantly lower than that of chronic cervicitis tissue. HPV16 L1 staining was mainly found in the cytoplasm of cervicitis tissue cells, and appeared brown or brown-yellow and granular. The majority of cervical cancer cells appeared light yellow, or unstained. Furthermore, the expression of HPV16 L1 decreased with increasing pathological grade.

In 48 cervical cancer samples, 27.1\% (13/48) of the tissue was positive for HPV16 L1 expression, and in the 12 cases of CIN III tissue, $41.7 \%$ (5/12) of tissue was positively stained. In 29 CIN I and CIN II samples, and in 30 chronic cervicitis samples, the tissue was strongly positively stained at a rate of $100.0 \%$ (supplementary Table 3). Results showed that the expression difference of HPV16 L1 protein in cervical cancer, CIN, and chronic cervicitis samples were statistically significant. Pathological diagnosis and HPV16 L1 protein expression were compared in chronic cervicitis vs. CIN III, chronic cervicitis vs. cervical cancer; CIN I and II vs. CIN III, and CIN I and II vs. cervical cancer $(P<0.05 ;)$.

Assessment of eEF1A2 and PI3K-Akt signal transduction pathway-related genes in cervical tissues

2.1 Real-time quantitative PCR to detect gene expression levels

The mRNA copy number of eEF1A2, PIK3CA, and Akt1 was significantly higher in cervical cancer than that in normal cervical tissue $(P<0.001$; fig.2, supplementary Table 4$)$.

2.2 General characteristics of patients

The positive rates of eEF1A2 protein expression in cervical cancer tissues of pathological grades 
were statistically significant $(P<0.05$; Table 2$)$.

2.3 Immunohistochemical detection of protein expression in cervical tissues

2.3.1 Expression of eEF1A2, PI3K, and p-Akt protein in cervical carcinoma and chronic cervicitis. Immunohistochemistry results showed that the expression intensity of eEF1A2 protein in cervical carcinoma was significantly higher than that in chronic cervicitis. Staining was mainly distributed in the cytoplasm of cancer cells, and appeared granular and brown or brown-yellow. The majority of chronic cervicitis tissues appeared light yellow or unstained. Furthermore, the expression of eEF1A2 protein increased with increased pathological grade. The expression of p-Akt and PI3K protein in cervical cancer was consistent with the expression of eEF1A2 protein, and was also distributed in the cytoplasm of cancer cells as brown-yellow particles. In addition, p-Akt and PI3K was more highly expressed in cervical cancer than in CIN tissue, while the expression in CIN was higher than that of chronic cervicitis (Fig. 3).

\subsubsection{Immunohistochemical analysis of eEF1A2, PI3K, p-Akt protein}

In the 58 cervical cancer samples assessed, $46.6 \%$ of the tissue was strongly positively stained for eEF1A2. The rate of positive staining in $41 \mathrm{CIN}$ tissue samples increased as a function of pathological grade. In 33 chronic cervicitis samples, the rate of strong positive staining was $21.2 \%$ (Fig. 4, supplementary Table 5). Experimental results showed that the expression of eEF1A2 protein in the cervical cancer, CIN, and chronic cervicitis tissue groups was significantly different. According to pathological diagnosis and eEF1A2 protein expression, following comparisons were made: chronic cervicitis vs. CIN I ( $\left.\chi^{2}=18.547, P<0.05\right)$; chronic cervicitis vs. cervical cancer $\left(\chi^{2}=6.112, P<0.05\right)$; CIN I vs. CIN II $\left(\chi^{2}=12.831, P<0.05\right)$; CIN II vs. CIN III $\left(\chi^{2}=6.075, P\right.$ $<0.05)$; and CIN I vs. cervical cancer $\left(\chi^{2}=8.225, P<0.05\right)$. The differences between chronic 
cervicitis vs. CIN II, chronic cervicitis vs. CIN III, CIN II vs. CIN III, CIN II vs. cervical cancer, and CIN III vs. cervical cancer were not statistically significant, $P>0.05$.

In the 47 cervical cancer samples, $31.9 \%$ of the tissue was PI3K strongly positive. In the $37 \mathrm{CIN}$ samples, the rate of positive staining increased as a function of pathological grade. In the 19 chronic cervicitis samples, strongly positive staining was observed in $21.1 \%$ of the tissue (Fig. 4, supplementary Table 6). Experimental results showed that the expression of PI3K in the cervical cancer, CIN, and chronic cervicitis groups was significantly different. According to pathological diagnosis and PI3K protein expression, following comparisons were made: chronic cervicitis vs. CIN I $\left(\chi^{2}=19.939, P<0.05\right)$; chronic cervicitis vs. CIN III $\left(\chi^{2}=8.786, P<0.05\right)$; chronic cervicitis vs. cervical cancer $\left(\chi^{2}=9.279, P<0.05\right)$; CIN I vs. CIN II $\left(\chi^{2}=6.589, P<0.05\right)$; and CIN I vs. cervical cancer $\left(\chi^{2}=11.301, P<0.05\right)$.

In the 37 cervical cancer samples, $62.2 \%$ of tissue was strongly positively stained for p-Akt. In the $41 \mathrm{CIN}$ samples, the rate of positive staining increased as a function of pathological grade. In the 18 chronic cervicitis samples, $16.7 \%$ of tissue was strongly positively stained for p-Akt (Fig. 4, supplementary Table 7). Experimental results showed that the expression of p-Akt protein in the cervical cancer, CIN, and chronic cervicitis groups was statistically significant. According to pathological diagnosis and p-Akt protein expression, following comparisons were made: chronic cervicitis vs. CIN I ( $\left.\chi^{2}=6.393, P<0.05\right)$; chronic cervicitis vs. cervical cancer $\left(\chi^{2}=11.24, P<\right.$ 0.05); CIN I vs. CIN III ( $\left.\chi^{2}=8.505, P<0.014\right)$; CIN I vs. cervical cancer $\left(\chi^{2}=18.248, P<0.05\right)$; CIN II vs. CIN III ( $\left.\chi^{2}=6.078, P<0.05\right)$; and CIN II vs. cervical cancer $\left(\chi^{2}=14.584, P<0.05\right)$.

\subsection{Protein expression in cervical cancer cell lines}

The expression of eEF1A2, PI3K, and p-Akt protein in HeLa, SiHa, and normal human umbilical 
vein endothelial cells was detected by western blot. Results showed that the expression of eEF1A2, $\mathrm{PI} 3 \mathrm{~K}$ and p-Akt protein in cervical cancer cells was significantly higher than in human umbilical vein endothelial cells ( $\mathrm{n}=3$ independent experiments, $P<0.05$; Fig. 5, supplementary Table 8 ).

\subsection{Suppression of eEF1A2 inhibits proliferation in cervical cancer cells}

In order to further explore the role of eEF1A2 in cervical cancer, we silenced the expression of eEF1A2 in SiHa and HeLa cells using siRNA (Fig. 6a). The effect of siRNA function was studied at protein levels. We then applied control-siRNA (si-NC) or eEF1A2-siRNA (si-eEF1A2) to the two groups of cervical cancer cells. The si-eEF1A2-treated cells showed a significantly slower rate of proliferation than the control group at 24,48 , and $72 \mathrm{~h}$ post-transfection (Fig. $6 \mathrm{~b}$ ). The addition of si-eEF1A2 also significantly inhibited colony formation (Fig. 6c). In summary, our data reveal that inhibiting eEF1A2 inhibits the growth of cervical cancer cells.

\section{Discussion}

In recent years, studies have shown that eEF1A2 is closely related to tumorigenesis via its ability to inhibit cellular apoptosis. It has been reported that the eEF1A2 gene is linked to the occurrence and development of different cancers, such as ovarian[9], gastric cancer[10], liver[11], and prostate cancer[12]. However, there are few studies concerning the role of eEF1A2 in cervical cancer. Although high-risk HPV16 infection has been identified as a key factor that induces cervical cancer, whether eEF1A2 plays a role in the development of cervical cancer remains unclear. Therefore, this study was conducted in order to assess the expression of eEF1A2 and members of the PI3K-Akt signal transduction pathway in cervical cancer, in order to determine its potential role in the development and progression of cervical cancer.

\section{The metabolic pathway of HPV16 L1}


HPV belongs to the family of papilloma DNA viruses, which are able to force normal cells to undergo continuous mitosis. It is a highly species-specific and special epitheliotropic virus whose DNA integrates into and exists within host cells. Under normal, healthy conditions, the body kills infected cells to prevent the replication of new viral particles, so as to resist viral infection. However, HPV can selectively stimulate cellular proliferation in order to circumvent the body's immune system. Direct or indirect stimulation of cellular proliferation may eventually lead to tumorigenesis[13, 14].

The main sexually-transmitted and pathogenic HPV variants are HPV6, 11, 16, 18. Among them, HPV16 and 18 are most highly related to the occurrence of cervical cancer[15-18].

HPV16 is a circular double-stranded DNA virus in which two late genes, L1 and L2, encode the structural proteins which form the outer shell of the virus. In the case of HPV16 viral infection leading to tumorigenesis, the HPV16 L1 protein is not detected in tumor tissue. Mechanistically, the expression of HPV16 L1 protein is related to the process of squamous epithelial cell differentiation. When squamous epithelial cells begin to differentiate, HPV16 completes its life cycle, leading to L1 protein expression[19, 20]. In tumor tissues, as a result of tissue de-differentiation, the virus is unable to initiate the expression of late protein programs, and the expression of HPV16 L1 protein can therefore not be detected.

In this study, the expression of HPV16 L1 protein in chronic cervicitis, CIN, and cervical cancer was examined by immunohistochemistry. Results showed that HPV16 L1 protein was expressed in chronic cervicitis, but weak or almost entirely absent in cervical cancer tissue. This result is consistent with the L1 viral shell protein decomposition and synthesis process described above. HPV16 L1 protein can be detected in early viral infections, and in this situation, 
the body's immune system may have identified the presence of HPV16 virus and mounted an immune response. According to our immunohistochemistry results, HPV16 L1 protein expression is decreased in cervical carcinoma tissue, and is further significantly decreased in invasive cervical cancer tissue. HPV16 L1 may therefore be considered as a diagnostic marker of CIN II and CIN III or CIN II and cervical cancer.

\section{The metabolic pathway of eEF1A2 and its expression in cervical carcinoma}

When the wild-type human eEF1A2 gene is transfected into mammalian cells, it is sufficient to promote independent cellular growth as well as enhance tumor growth in nude mice. This indicates that eEF1A2 plays an important role in tumor occurrence and development. However, the precise mechanisms by which eEF1A2 contributes to carcinogenesis is not clear. Nisha et al. ${ }^{10}$ transfected exogenous eEF1A2 into NIH3T3 mouse fibroblasts, and found that eEF1A2 significantly increased the growth of cellular clones compared to control transfected cells. Furthermore, when cellular clones were injected into nude mice, tumors formed after 3 weeks. These results suggest that eEF1A2 may act as an oncogene. We extracted RNA from 20 cervical tissue samples (including 10 cases of cervical cancer and 10 cases of normal cervical tissue), and used qRT-PCR to detect eEF1A2 mRNA expression. Statistical analysis showed that expression in the cervical cancer group was significantly higher than in the normal cervical group. These results suggests that an increase in eEF1A2 mRNA expression leads to the occurrence of cervical cancer. It has also been reported that eEF1A2 mRNA expression in breast[21], liver[22], prostate[13, 23] cancer is significantly higher than in normal tissues, and is thought to play an important role in tumor cell proliferation, metastasis and invasion.

Studies have shown that the abnormal expression of eEF1A2 can not only lead to uncontrolled 
proliferation and apoptosis of tumor cells, but also play an important role in the process of tumor invasion and metastasis. Edmonds et al.[24] have found that the expression levels of eEF1A2 in rat breast cancer cells with metastatic potential is significantly higher than that of non-metastatic cells. In our study, the expression of eEF1A2 protein and the rate of strongly positive eEF1A2 expression in cervical cancer tissue was significantly higher than that in chronic cervicitis tissue. According to the positive rate of eEF1A2 expression in cervical cancer, we compared pathological grade of patients, and found that the different pathological grades of cervical cancer differed significantly in their level of eEF1A2 protein expression: with a decline in the degree of differentiation of cervical cancer, the expression of eEF1A2 increased. The above experimental results are consistent with the results of previous studies. This indicates that eEF1A2 may play an important role in the occurrence and development of cervical cancer.

\section{PI3K-Akt signal transduction pathway and cervical cancer}

The PI3K-Akt pathway can be activated in two ways. Firstly, via interactions with a growth factor receptor with a phosphorylated tyrosine residue and a connexin, the conformation of the dimer is altered, leading to activation. Secondly, when PI3K combines with p110 $\alpha$, PI3K is activated in a Ras-dependent manner[25].

The role of the $\mathrm{P} 13 \mathrm{~K}$ p110 $\alpha$ protein is to promote cellular proliferation and inhibit apoptosis under normal physiological conditions[26]. When the PIK3CA gene is mutated, the levels of PI3K $\mathrm{p} 110 \alpha$ protein are increased, leading to increased activity of P13K. Through the PI3K-Akt signaling pathway, Akt is excessively activated, which leads to excessive cellular proliferation, and also affects differentiation and inhibits apoptosis, contributing to tumorigenesis[27]. In this study, the changes in mRNA expression levels of PIK3CA and Akt1 were assessed using qRT-PCR 
in cervical cancer tissue. Results showed that the expression of mRNA in cervical carcinoma and normal cervical tissues was higher than that in normal cervical tissues. At the same time, we also detected the expression of the PIK3CA protein product P13K p110 $\alpha$ and the product of activated Akt, p-Akt, in chronic cervicitis, CIN I, CIN II, CIN III, and cervical cancer tissue. Results showed that PIK3CA is involved in the entire process of the development of cervical cancer, which indicates that the PI3K-Akt signal transduction pathway may play an important role in the occurrence and development of cervical cancer.

Previous studies have shown that the PI3K-Akt signaling pathway plays an important role in cancer onset and progression[28]. We also detected the expression of PI3K, p-Akt, and eEF1A2 in cervical cancer cell lines and normal human epithelial cells by western blot. Results showed that the expression of PI3K, p-Akt, and eEF1A2 in cervical cancer cells was higher than in normal control cells.

In summary, our results suggest that the levels of eEF1A2, PI3K, and Akt1 mRNA in cervical tissue is higher than in normal cervical tissue. At the level of protein expression, cervical cancer tissue had significantly higher levels than chronic cervicitis, and there was a significant difference between the rate of positive eEF1A2 expression in cervical cancer tissue and pathological grade. These results indicate that abnormally high expression of genes in the PI3K-Akt signaling pathway, as well as eEF1A2, may play an important role in the occurrence of cervical cancer. These results lay the foundation for future research aiming to prevent and treat cervical cancer. At the same time, these results including mRNA and protein levels provide a reference value for the diagnosis and pathological grading of cervical cancer. Our assessment of the expression of HPV16 L1, eEF1A2, and other PI3K-Akt signal transduction pathway genes in cervical cancer provides critical 
information that will contribute to an understanding of the occurrence and development of cervical carcinoma.

\section{Conclusion}

HPV16 L1 protein expression was reduced in situ of cervical carcinoma, and was further decreased in invasive cervical cancer tissues. HPV16 L1 expression may therefore be used as a diagnostic marker of CIN II, CIN III, or cervical cancer. The expression of eEF1A2, PI3K and Akt genes may also play a role in the tumorigenesis and development of cervical cancer. Furthermore, eEF1A2 may act via the PI3K-Akt signal transduction pathway.

\section{Abbreviations:}

eEF1A2:eukaryotic translation elongation factor 1 alpha 2; HPV: Human papillomavirus; CIN: cervical intraepithelial neoplasia

\section{Acknowledgements}

We acknowledge that this work was supported in part by the National Natural Science Foundation of China (grant number: 82060518, U1503125);

International Science and Technology Collaboration Projector of Xinjiang Production and Construction Corps (grant number : 2019BC007).

\section{Author Contributions}

HZX and HP made main contributions to the document, designed the research, and participated in specimen collection, sorting data, data analysis and drafting the manuscript; XYZ, CHZ assisted in specimen collection; HTL and WNZ were mainly responsible for guiding data analysis; DML, ZMP and RFS provided substantial guidance, supported help in modifying article and revised the manuscript. All authors read and approved the final manuscript.

\section{Funding}


This work was supported in part by the National Natural Science Foundation of China (grant number: 82060518, U1503125);

International Science and Technology Collaboration Projector of Xinjiang Production and Construction Corps (grant number : 2019BC007).

\section{Availability of data and materials}

The datasets used and/or analyzed during the current study are available from the corresponding author on reasonable request. For the corresponding author, please contact Professor Zemin Pan, at panteacher89@sina.com.

\section{Ethics approval and consent to participate}

The sample collection for this study was approved by the Ethical Committee of the First Affiliated Hospital of the Medical College of Shihezi University, and informed consent was obtained from all of the participants.

\section{Consent for publication}

Not applicable.

\section{Competing interests}

The authors declare that they have no competing interests.

\section{Author details}

${ }^{1}$ Department of Biochemistry and Molecular Biology, School of Medicine, Shihezi University, Xinjiang Endemic and Ethnic Disease and Education Ministry Key Laboratory, Shihezi, Xinjiang, 832002, China

${ }^{2}$ Department of Anatomy and Histology and Embryology, Development and Regeneration Key Lab of Sichuan Province, Chengdu Medical College, Chengdu, Sichuan, 610000, China

${ }^{3}$ School of Science and Engineering, Genecology Research Centre, University of the Sunshine Coast, Sippy Downs, Queensland, 4556, Australia

\section{References}


1. Yi, Y., et al., Assessing the Effectiveness of a Cervical Cancer Screening Program in a Hospital-based Study. Biomedical \& Environmental Sciences, 2015. 28(1): p. 80-84.

2. Thones, N. and M. Muller, Oral immunization with different assembly forms of the HPV 16 major capsid protein L1 induces neutralizing antibodies and cytotoxic T-lymphocytes. Virology, 2007. 369(2): p. 375-88.

3. Pan, Z., et al., Differential gene expression identified in Uigur women cervical squamous cell carcinoma by suppression subtractive hybridization. Neoplasma, 2010. 57(2): p. 123-8.

4. Amiri, A., ., et al., eEF1A2 activates Akt and stimulates Akt-dependent actin remodeling, invasion and migration. Oncogene, 2007. 26(21): p. 3027-3040.

5. $\quad$ Pecorari, L., et al., Elongation Factor 1 alpha interacts with phospho-Akt in breast cancer cells and regulates their proliferation, survival and motility. Molecular Cancer,8,1(2009-08-03), 2009. 8(1): p. 58.

6. Tianyan, G., F. Frank, and A.C. Newton, PHLPP: a phosphatase that directly dephosphorylates Akt, promotes apoptosis, and suppresses tumor growth. Molecular Cell, 2005. 18(1): p. 13-24.

7. Ward, S.G. and F. Peter, Isoform-specific phosphoinositide 3-kinase inhibitors as therapeutic agents. Current Opinion in Pharmacology, 2003. 3(4): p. 426-434.

8. $\quad$ Wen, Z., et al., Minimally invasive percutaneous nephrolithotomy with multiple mini tracts in a single session in treating staghorn calculi. Urological Research, 2011. 39(2): p. 117-122.

9. Anand, N., et al., Protein elongation factor EEF1A2 is a putative oncogene in ovarian cancer. Nat Genet, 2002. 31(3): p. 301-5.

10. Yang, S., et al., Overexpression of eukaryotic elongation factor 1 alpha-2 is associated with poorer prognosis in patients with gastric cancer. J Cancer Res Clin Oncol, 2015. 141(7): p. 1265-75.

11. Schlaeger, C., et al., Etiology-dependent molecular mechanisms in human hepatocarcinogenesis. Hepatology, 2008. 47(2): p. 511-20.

12. Longerich, T., EEF1A2 inhibits the p53 function in hepatocellular carcinoma via PI3K/AKT/mTOR-dependent stabilization of MDM4. Pathologe, 2014. 35: p. 177-184.

13. Sun, Y., et al., Up-regulation of eEF1A2 promotes proliferation and inhibits apoptosis in prostate cancer. Biochem Biophys Res Commun, 2014. 450(1): p. 1-6.

14. Demers, G.W., et al., Growth arrest by induction of p53 in DNA damaged keratinocytes is bypassed by human papillomavirus 16 E7. Proc Natl Acad Sci U S A, 1994. 91(10): p. 4382-6.

15. Jones, D.L. and K. Münger, . Analysis of the p53-mediated G1 growth arrest pathway in cells expressing the human papillomavirus type 16 E7 oncoprotein. Journal of Virology, 1997. 71(4): p. 2905-2912.

16. Walboomers, J.M., et al., Human papillomavirus is a necessary cause of invasive cervical cancer worldwide. J Pathol, 1999. 189(1): p. 12-9.

17. Bosch, F.X., et al., The causal relation between human papillomavirus and cervical cancer. $\mathbf{J}$ Clin Pathol, 2002. 55(4): p. 244-65.

18. Steenbergen, R.D., et al., HPV-mediated transformation of the anogenital tract. J Clin Virol, 2005. 32 Suppl 1: p. S25-33.

19. Peto, J., et al., The cervical cancer epidemic that screening has prevented in the UK. Lancet, 2004. 364(9430): p. 249-56. 
20. Doorbar, J., et al., The biology and life-cycle of human papillomaviruses. Vaccine, 2012. 30 Suppl 5: p. F55-70.

21. Giudici, F., et al., Elevated levels of eEF1A2 protein expression in triple negative breast cancer relate with poor prognosis. 2019. 14(6): p. e0218030.

22. Yang, G. and H. Zong, Overexpression of PDZK1IP1, EEF1A2 and RPL41 genes in intrahepatic cholangiocarcinoma. Mol Med Rep, 2016. 13(6): p. 4786-90.

23. Worst, T.S., et al., The EEF1A2 gene expression as risk predictor in localized prostate cancer. BMC Urol, 2017. 17(1): p. 86.

24. Edmonds, B.T., et al., Elongation factor-1 alpha is an overexpressed actin binding protein in metastatic rat mammary adenocarcinoma. J Cell Sci, 1996. 109 ( Pt 11): p. 2705-14.

25. Osaki, M., M. Oshimura, and H. Ito, PI3K-Akt pathway: its functions and alterations in human cancer. Apoptosis, 2004. 9(6): p. 667-76.

26. Cantley, L.C., The Phosphoinositide 3-Kinase Pathway. Science, 2002. 296(5573): p. 1655-7.

27. Castaneda, C.A., et al., The phosphatidyl inositol 3-kinase/AKT signaling pathway in breast cancer. Cancer Metastasis Rev, 2010. 29(4): p. 751-9.

28. Martini, M., et al., PI3K/AKT signaling pathway and cancer: an updated review. Ann Med, 2014. 46(6): p. 372-83.

\section{Figure legends}

Fig. 1. Expression of HPV16 L1 protein in chronic cervicitis, cervical intraepithelial neoplasia, and cervical carcinoma $(\mathrm{SP} \times 200)$. A. Chronic cervicitis. B. CIN I. C. CIN II. D. CIN III. E. Cervical carcinoma.

Fig. 2. Expression of eEF1A2, PIK3CA, and Akt1 mRNA in cervical cancer and normal cervical tissues. The expression of mRNA in cervical cancer was higher than that in normal cervical tissues.

Fig. 3. Expression of eEF1A2, PI3K, and p-Akt protein in different cervical tissues $(\mathrm{SP} \times 100)$. The expression of eEF1A2 protein is shown in A, B, and C. The expression of PI3K protein is shown in $\mathrm{D}, \mathrm{E}$, and $\mathrm{F}$. The expression of p-Akt protein is shown in $\mathrm{G}, \mathrm{H}$, and $\mathrm{I}$. A, D, and $\mathrm{G}$ are chronic cervicitis tissue. B, E, and $\mathrm{H}$ are cervical intraepithelial neoplasia tissue. C, F, and I are cervical carcinoma tissue.

Fig. 4. Expression of eEF1A2, PI3K, p-Akt protein in chronic cervicitis, cervical intraepithelial neoplasia (CIN I, CIN II, CIN III ), and cervical carcinoma.

Fig. 5. The expression of eEF1A2, PI3K and p-Akt protein in cervical cancer cell lines and normal epithelial cells. The samples are from the same experiment, and the gel/blotting are processed in 
parallel.

Fig. 6. Knockdown of eEF1A2 significantly inhibits the proliferation of cervical cancer cells. a. The efficiency of si-eEF1A2 was confirmed by western blot in SiHa and HeLa cells. b. Viable cell numbers were detected by CCK- 8 assays after transfection for 24,48 , and $72 \mathrm{~h}$ in cervical cancer cells. c. Transfected cells were seeded in 6-well plated and incubated for 2 weeks. The colonies formed in si-eEF1A2 transfected cells were fewer in number and smaller in size. Data are presented as the mean and standard deviation of three experimental replicates. Representative images are shown. $(* P<0.05, * * P<0.01)$. The samples are from the same experiment, and the gel/blotting are processed in parallel. 


\section{Figures}

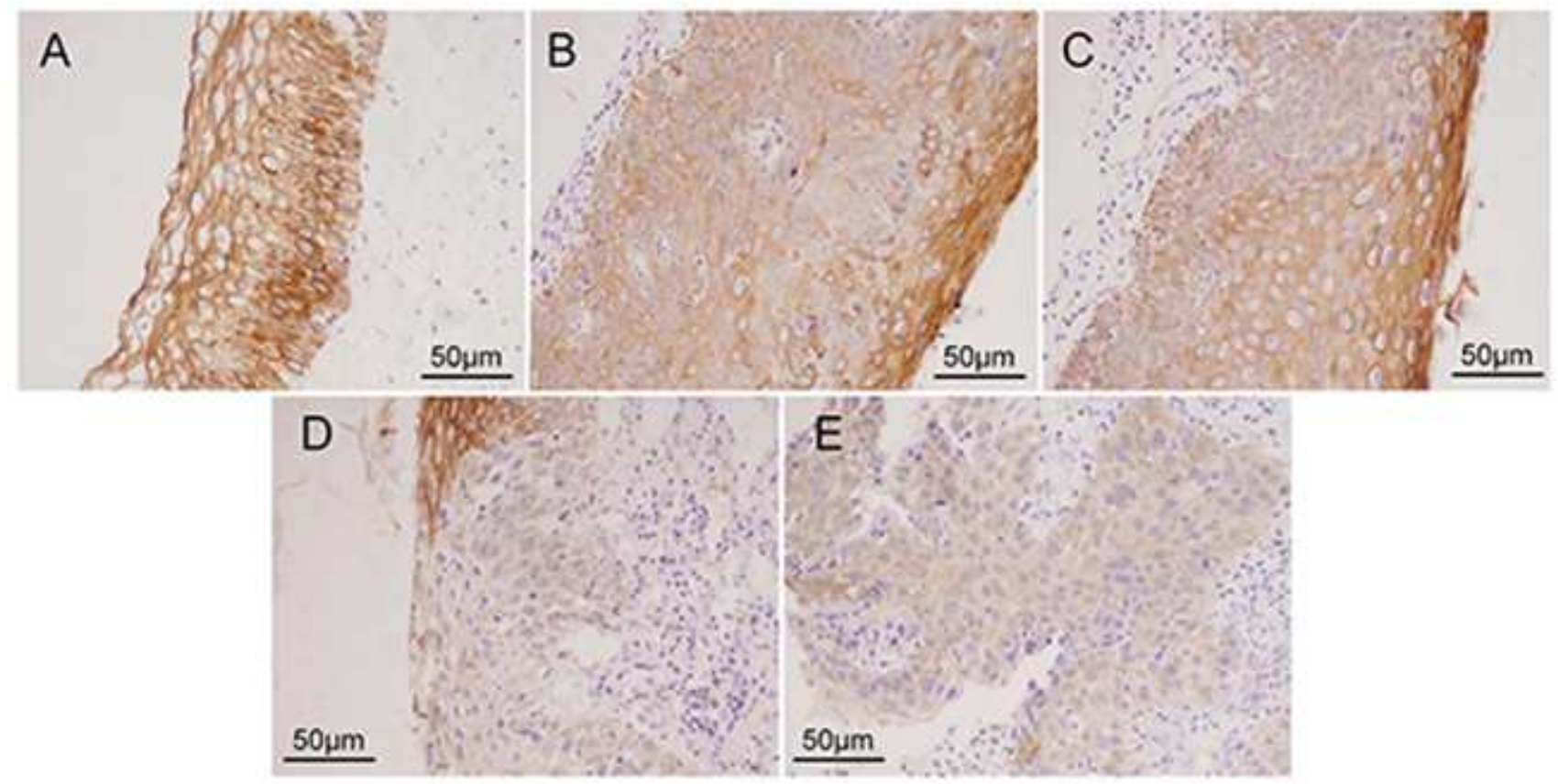

\section{Figure 1}

Expression of HPV16 L1 protein in chronic cervicitis, cervical intraepithelial neoplasia, and cervical carcinoma (SP×200). A. Chronic cervicitis. B. CIN I. C. CIN II. D. CIN III. E. Cervical carcinoma. 


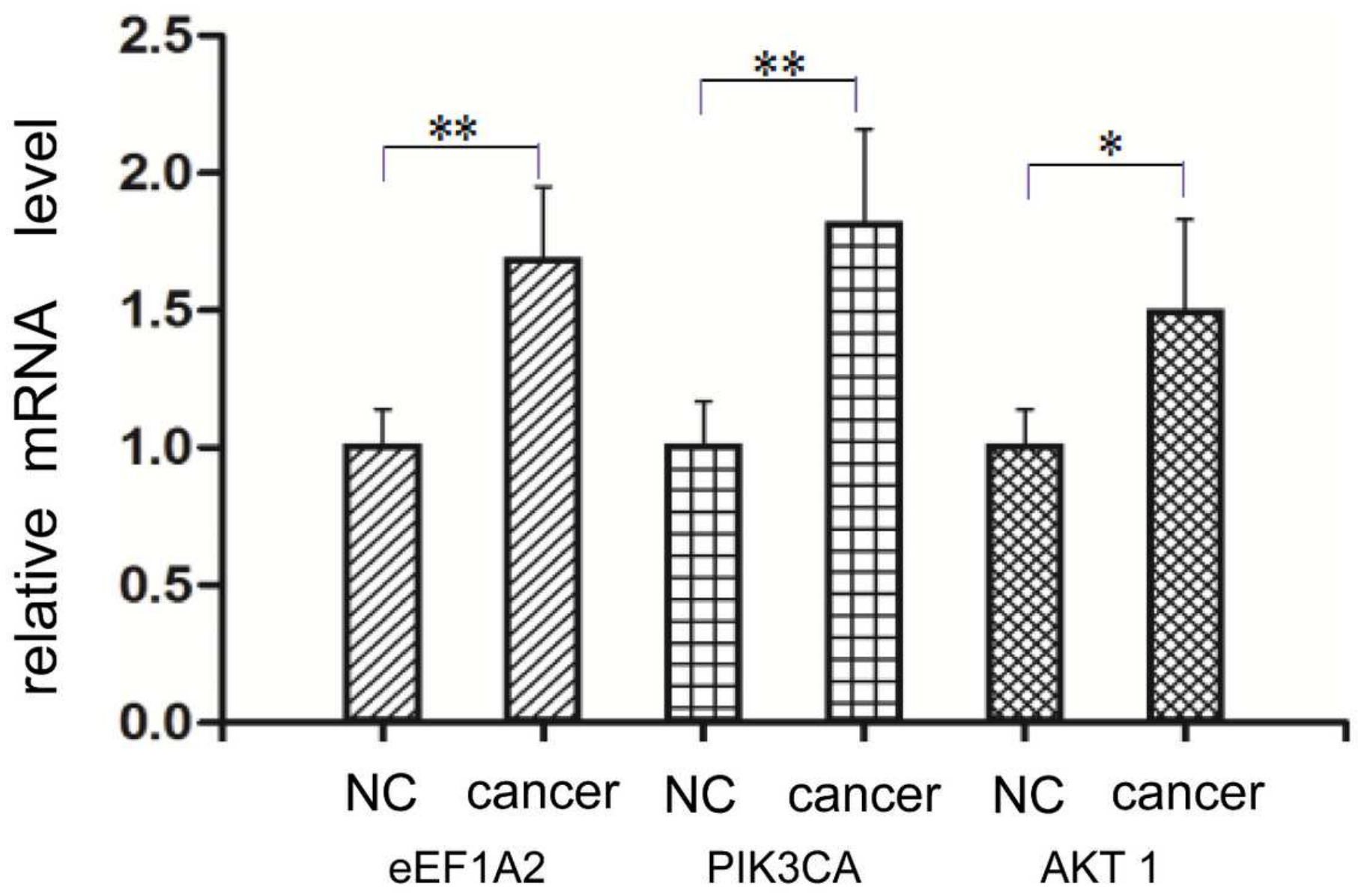

Figure 2

Expression of eEF1A2, PIK3CA, and Akt1 mRNA in cervical cancer and normal cervical tissues. The expression of mRNA in cervical cancer was higher than that in normal cervical tissues. 


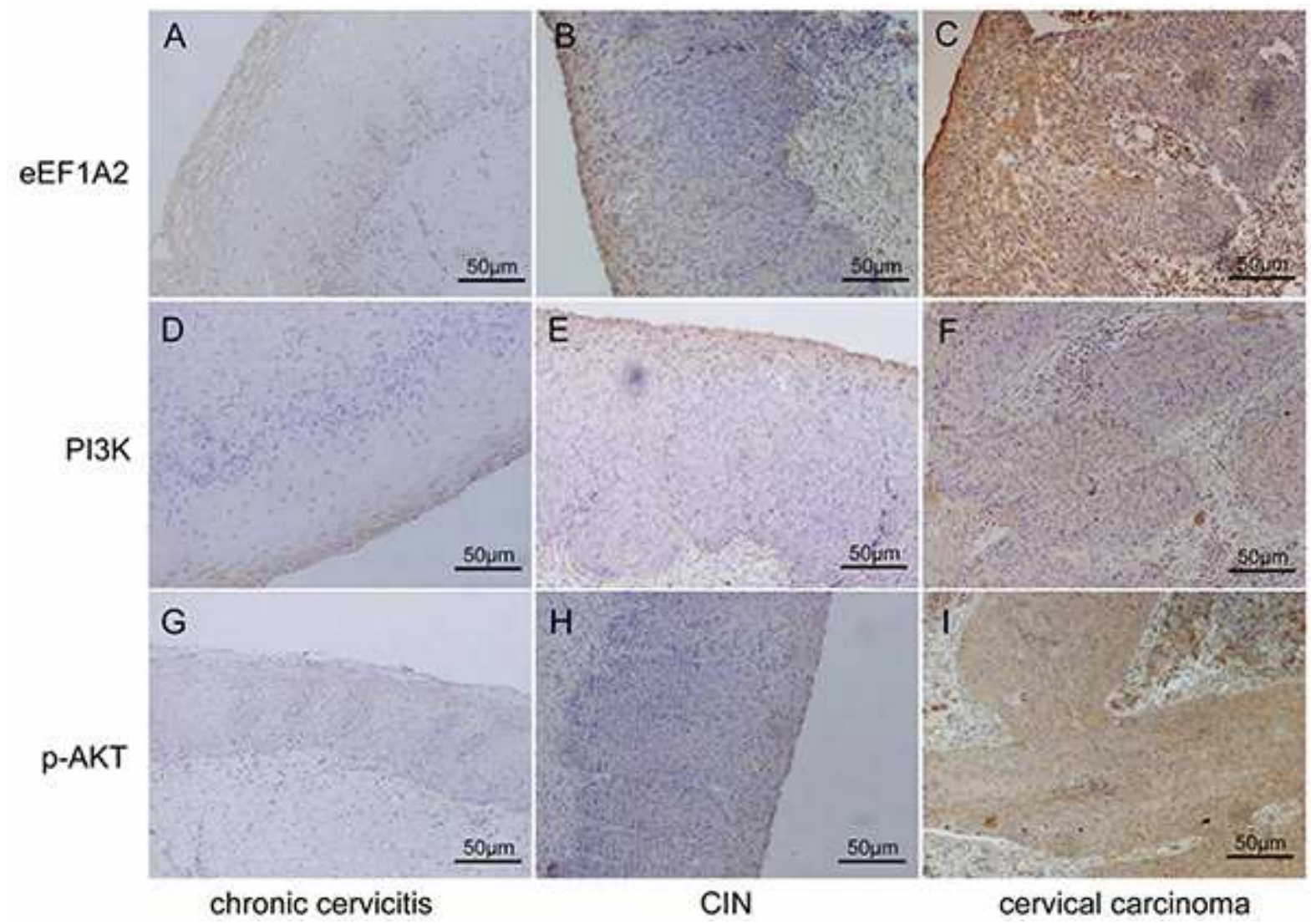

Figure 3

Expression of eEF1A2, PI3K, and p-Akt protein in different cervical tissues (SP×100). The expression of eEF1A2 protein is shown in $A, B$, and $C$. The expression of PI3K protein is shown in $D, E$, and $F$. The expression of p-Akt protein is shown in $G, H$, and I. A, D, and $G$ are chronic cervicitis tissue. $B, E$, and $H$ are cervical intraepithelial neoplasia tissue. C, F, and I are cervical carcinoma tissue.

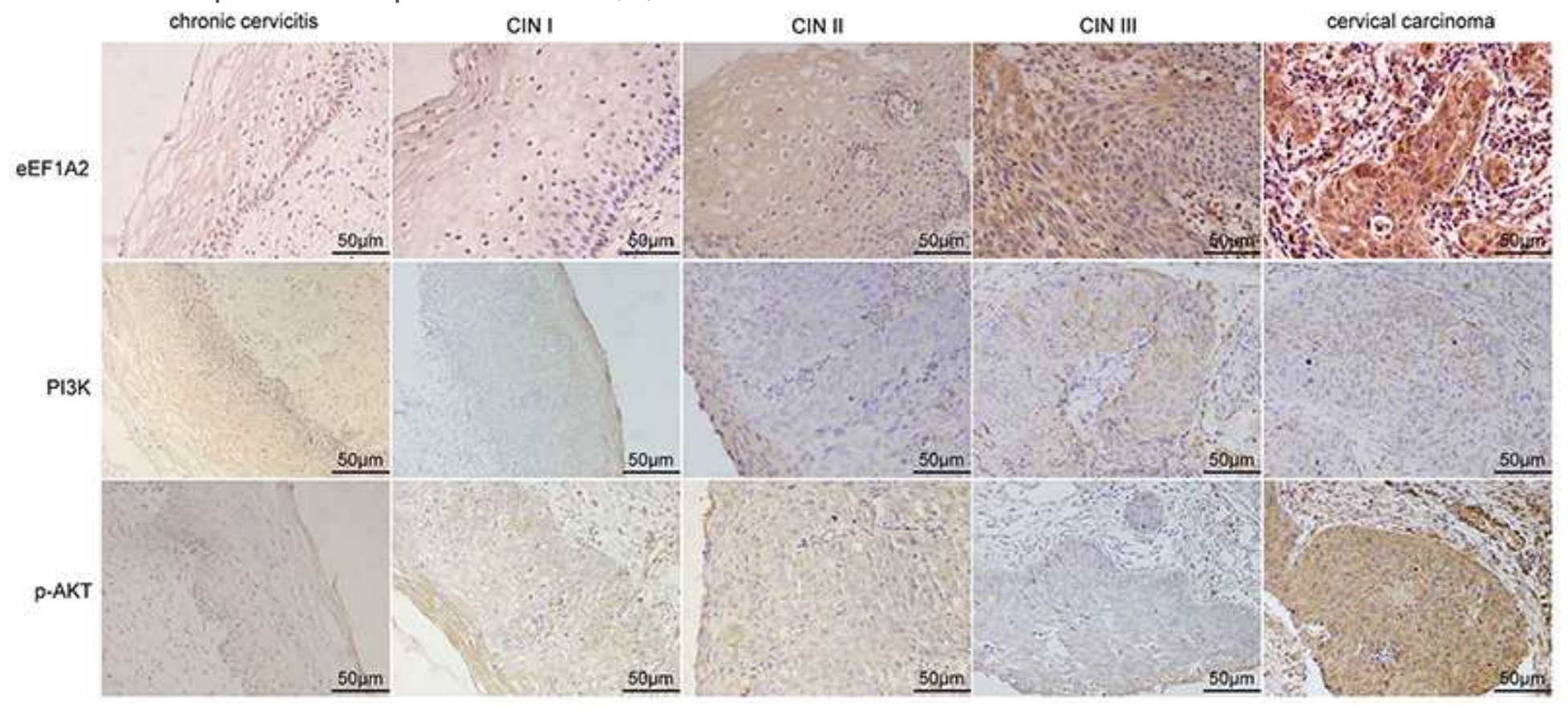

Figure 4 
Expression of eEF1A2, PI3K, p-Akt protein in chronic cervicitis, cervical intraepithelial neoplasia (CIN I, CIN II, CIN III ) , and cervical carcinoma.
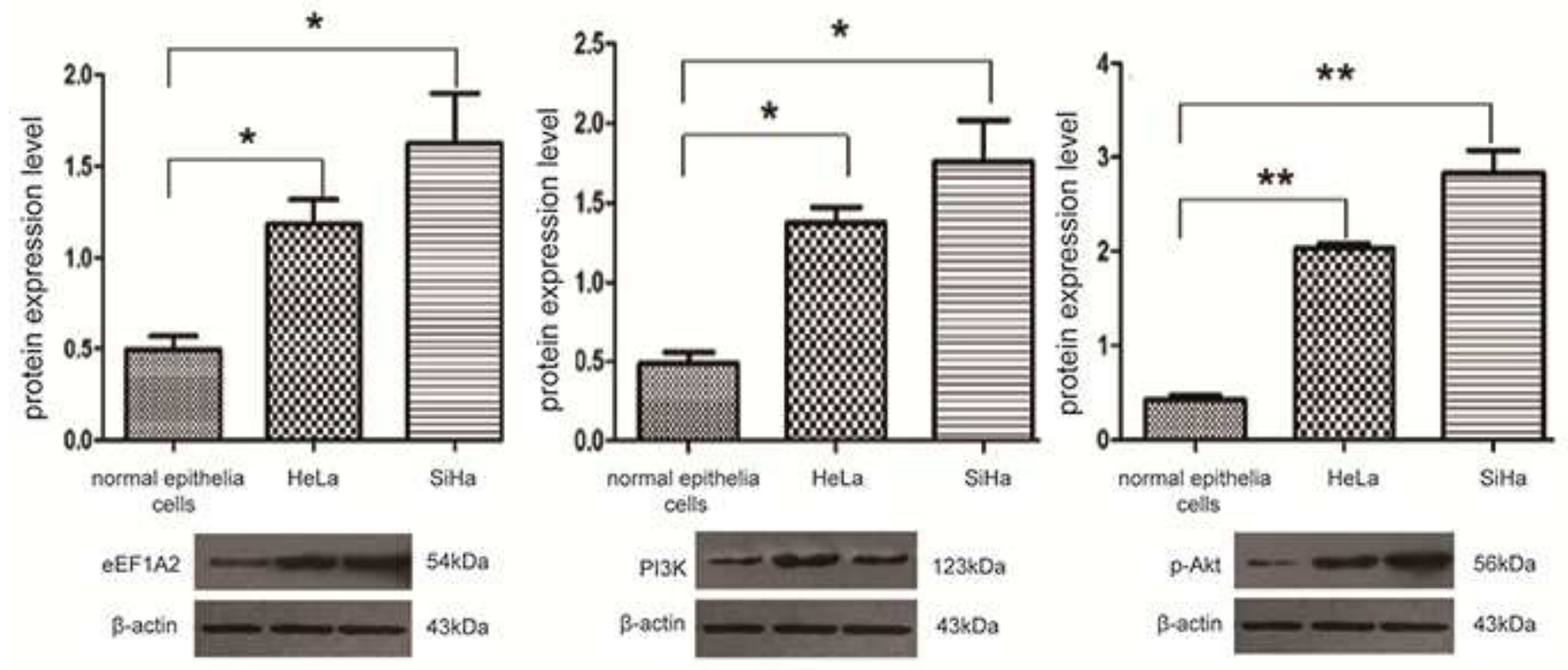

\section{Figure 5}

The expression of eEF1A2, PI3K and p-Akt protein in cervical cancer cell lines and normal epithelial cells. The samples are from the same experiment, and the gel/blotting are processed in parallel.

a

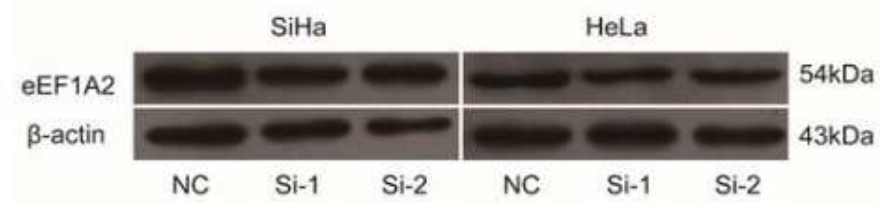

b
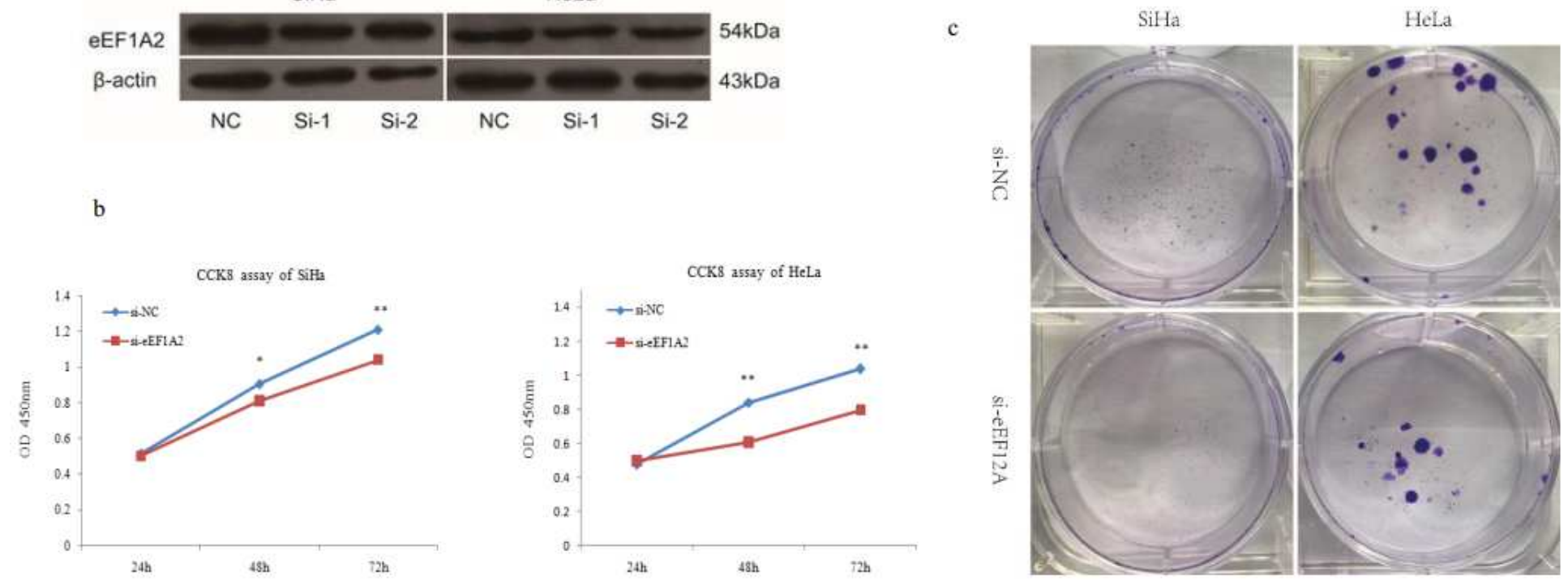

Figure 6

Knockdown of eEF1A2 significantly inhibits the proliferation of cervical cancer cells. a. The efficiency of si-eEF1A2 was confirmed by western blot in SiHa and HeLa cells. b. Viable cell numbers were detected by CCK-8 assays after transfection for 24,48 , and $72 \mathrm{~h}$ in cervical cancer cells. c. Transfected cells were seeded in 6-well plated and incubated for 2 weeks. The colonies formed in si-eEF1A2 transfected cells were fewer in number and smaller in size. Data are presented as the mean and standard deviation of 
three experimental replicates. Representative images are shown. ( $\left.{ }^{*}<0.05, * \star P<0.01\right)$. The samples are from the same experiment, and the gel/blotting are processed in parallel.

\section{Supplementary Files}

This is a list of supplementary files associated with this preprint. Click to download.

- Supplementaryinformation.docx 